\title{
Effect of intrinsic rotations, microstructural expansion and contractions in initial boundary value problem of thermoelastic bodies
}

\author{
Marin I Marin ${ }^{1 *}$, Ravi P Agarwal ${ }^{2}$ and Ibrahim A Abbas ${ }^{3,4}$
}

${ }^{*}$ Correspondence:

m.marin@unitbv.ro

${ }^{1}$ Department of Mathematics,

Transilvania University of Brasov, Brasov, Romania

Full list of author information is

available at the end of the article

\begin{abstract}
This study is dedicated to some basic theorems in the thermoelastodynamics of microstretch bodies. Our intention is to show that the presence of the microstretch does not affect the main characteristics of the mixed initial boundary value problem for thermoelastic bodies. The result regarding the uniqueness theorem is derived with no definiteness assumptions on the elastic coefficients and in the absence of the restriction that the conductivity tensor is positive definite. In the last part of the paper we establish a basic relation which leads to the reciprocal theorem and to another uniqueness result.
\end{abstract}

MSC: 35M30; 35Q74; 74A15; 74A60; 74M25

Keywords: thermoelastic; microstretch; seismic waves; earthquake

\section{Introduction}

The theory of micromorphic elastic solids was first elaborated by Eringen (see, for instance, [1]). Then Eringen has generalized [2] this theory in order to cover the theory of thermo-microstretch elastic materials. In short, this is a theory of thermoelasticity with microstructure that includes intrinsic rotations and microstructural expansion and contractions.

The micromorphic theory was introduced to describe adequately the behavior of materials such a liquid crystal, fluid suspensions, polycrystalline aggregates, and granular media. For this it is necessary to introduce into the continuum theory some terms reflecting the microstructure of the materials. In the context of this theory, each material point has three deformable directors.

A continuum body is a microstretch continuum if the directors are constrained to have only breathing-type microdeformations. All points of a microstretch continuum can stretch and contract independently of their translations and rotations.

This theory is expected to find applications in the treatment of composites materials reinforced with chopped fibers. Also, this theory can be useful in applications which deal with porous materials as geological materials, solid packed granular materials, and many others.

@2014 Marin et al.; licensee Springer. This is an Open Access article distributed under the terms of the Creative Commons Attribution License (http://creativecommons.org/licenses/by/2.0), which permits unrestricted use, distribution, and reproduction in any medium, provided the original work is properly cited. 
On the other hand, materials which operate at elevated temperatures will invariably be subjected to heat flow at some time during normal use. Such heat flow will involve a nonlinear temperature distribution, which will inevitable give rise to thermal stresses. For these reasons, the development, design, and selection of materials for high temperature applications require a great deal of care. The role of the pertinent material properties and other variables which can affect the magnitude of thermal stress must be considered.

The theory of microstretch elastic bodies is generalized from the micropolar theory introduced by Cosserat. There are many papers which are concerned with this theory. For instance, Ciarletta in [3] has used the basic results deduced by Eringen in order to investigate the isothermal bending of microstretch elastic plates. Ciarletta et al. dedicated the paper [4] to the study of some basic properties of wave numbers of the longitudinal and transverse plane harmonic waves, in the context of thermoelasticity for materials with voids.

In the paper [5] Iesan and Pompei have presented a solution of Boussinesq-SomiglianaGalerkin type for the boundary value problem in this context.

In the paper [6], Agarwal et al. presented new existence results for initial value problems. The nonlinearity may be singular in its dependent variable and is allowed to change sign. Also, the paper of Agarwal and O'Regan [7] presents existence result for some boundary value problems definite on infinite intervals, which, in particular, includes a problem which arises in the theory of colloids. In the studies [8-11] we tackle some questions with regards to the microstretch-thermoelastic materials. Thus, in the paper [9], we use the Lagrange identity to prove the uniqueness theorem and some continuous dependence theorems without recourse to any energy conservation law, or to any boundedness assumptions on the thermoelastic coefficients. Also, the paper [8] is concerned with microstretchthermoelastic materials. In this context, for the mixed initial boundary value problem, we prove that the Cesaro means of the kinetic and strain energies of a solution with finite energy become asymptotically equal as time tends to infinity. A model of microstretchthermoelastic body with two temperatures is presented in the paper [12].

The study [13] is dedicated to the study of propagation of shear waves in a nonhomogeneous anisotropic incompressible gravity field, and an initially stressed medium is studied.

The paper [14] investigates the longitudinal wave propagation in a perfectly conducting elastic circular cylinder in the presence of an axial initial magnetic field. Other results regarding generalized thermoelasticity can be found in the papers $[15,16]$.

The present paper must be considered as a first step toward a better understanding of microstretch and thermal stress in the study of the above enumerated materials.

The reciprocity and representation relations that appear in our study constitute powerful theoretical tools in the assessment of the theory of seismic-sources mechanism, in the studies connected with seismic wave propagation.

Also, we think that this paper is a good help to understanding the application of the microstretch mechanism to earthquake problems.

There are many results regarding the mechanism of earthquakes, as, for instance, in the papers [17] and [18] and in the references therein. 


\section{Basic equations}

For convenience the notations and terminology chosen are almost identical to those of our studies $[8,9]$. The present paper is concerned with an anisotropic and homogeneous material.

Let the body occupy, at time $t=0$, a properly regular region $B$ of the three-dimensional Euclidian space, bounded by the piece-wise smooth surface $\partial B$; and we denote the closure of $B$ by $\bar{B}$. We refer the motion of the body to a fixed system of rectangular Cartesian axes $O x_{i}, i=1,2,3$ and adopt the Cartesian tensor notation. Points in $B$ are denoted by $x_{j}$ and $t \in[0, \infty)$ is the temporal variable. Throughout this work the Einstein summation convention over repeated indices is used. The subscript $j$ after a comma indicates partial differentiation with respect to the spatial argument $x_{j}$. All Latin subscripts are understood to range over the integers $(1,2,3)$, while the Greek indices have the range $(1,2)$. A superposed dot denotes the derivatives with respect to the time variable $t$. Also, the spatial argument and the time argument of a function will be omitted when there is no likely confusion.

Let us denote by $u_{i}$ the components of the displacement vector and by $\varphi_{i}$ the components of the microrotation vector. Also, we denote by $\omega$ a scalar function that characterizes the microstretch and by $\theta$ the temperature measured from the constant absolute temperature $T_{0}$ of the body in its reference state.

As usual, we denote by $t_{i j}$ the components of the stress tensor and by $m_{i j}$ the components of the couple stress tensor over $B$. Also, we denote by $\lambda_{i}$ the components of the microstress vector.

In the dynamic theory of the thermoelasticity of microstretch bodies the fundamental system of field equations consists of:

- the equations of motion

$$
\begin{aligned}
& t_{j i, j}+F_{i}=\varrho \ddot{u}_{i}, \\
& m_{j i, j}+\varepsilon_{i j k} t_{j k}+G_{i}=I_{i j} \ddot{\varphi}_{j} ;
\end{aligned}
$$

- the balance of the equilibrated forces

$$
\lambda_{i, i}+L=J \ddot{\omega}
$$

- the energy equation

$$
T_{0} \dot{\eta}=q_{i, i}+S
$$

For an anisotropic and homogeneous microstretch thermoelastic material, the constitutive equations have the form

$$
\begin{aligned}
& t_{i j}=A_{i j m n} \varepsilon_{m n}+B_{i j m n} \mu_{m n}+D_{i j k} \gamma_{k}+a_{i j} \theta, \\
& m_{i j}=B_{m n i j} \varepsilon_{m n}+C_{i j m n} \mu_{m n}+E_{i j k} \gamma_{k}+b_{i j} \theta, \\
& \lambda_{i}=D_{m n i} \varepsilon_{m n}+E_{m n i} \mu_{m n}+F_{i j} \gamma_{j}+c_{i} \theta, \\
& \eta=-a_{i j} \varepsilon_{i j}-b_{i j} \mu_{i j}-c_{i} \gamma_{i}+d \theta, \\
& q_{i}=k_{i j} \theta_{j, j}
\end{aligned}
$$


where $A_{i j m n}, B_{i j m n}, C_{i j m n}, D_{i j k}, E_{i j k}, F_{i j}, a_{i j}, b_{i j}, c_{i}, d$, and $k_{i j}$ are the characteristic constitutive coefficients, and they obey the following symmetry relations:

$$
A_{i j m n}=A_{m n i j}, \quad C_{i j m n}=C_{m n i j}, \quad F_{i j}=F_{j i}, \quad k_{i j}=k_{j i} .
$$

In the above equations we have used the following notations:

- $F_{i}$ the components of body force;

- $G_{i}$ the components of body couple;

- $L$ the generalized external body load;

- $\varrho$ is the reference constant mass density;

- $J$ and $I_{i j}=I_{j i}$ are the coefficients of microinertia;

- $\lambda_{i}$ are the components of the internal hypertraction vector;

- $\eta$ is the entropy per unit mass;

- $S$ is the heat supply per unit mass;

- $q_{i}$ are the components of heat flux vector.

The components of the strain tensors $\varepsilon_{i j}, \mu_{i j}$, and $\gamma_{i}$ are defined by means of the geometric equations:

$$
\varepsilon_{i j}=u_{j, i}+\varepsilon_{j i k} \varphi_{k}, \quad \mu_{i j=} \varphi_{j, i}, \quad \gamma_{i}=\omega_{, i}
$$

where $\varepsilon_{i j k}$ is the alternating symbol.

The second law of thermodynamics implies that

$$
k_{i j} \xi_{i} \xi_{j} \geq 0, \quad \forall \xi_{i}
$$

that is, the conductivity tensor $\mathbf{k}$ is positive semi-definite only.

In what follows we need the following regularity assumptions:

(1) the constitutive coefficients are continuously differentiable functions on $\bar{B}$;

(2) $\varrho$ and $d$ are continuous functions on $\bar{B}$;

(3) $F_{i}, G_{i}$ and $L$ are continuous functions on $\bar{B} \times I$, where $I=[0, \infty)$.

We denote by $t_{i}$ the components of surface traction, $m_{i}$ the components of surface couple, $\lambda$ the microsurface traction and $q$ the heat flux. These quantities are defined by

$$
t_{i}=t_{j i} n_{j}, \quad m_{i}=m_{j i} n_{j}, \quad \lambda=\lambda_{i} n_{i}, \quad q=q_{i} n_{i},
$$

at regular points of the surface $\partial B$.

Here, $n_{i}$ are the components of the outward unit normal of the surface $\partial B$.

Along with the system of field equations (1)-(4) we consider the following initial conditions:

$$
\begin{array}{ll}
u_{i}(x, 0)=a_{i}^{0}(x), & \dot{u}_{i}(x, 0)=a_{i}^{1}(x), \\
\varphi_{i}(x, 0)=b_{i}^{0}(x), & \dot{\varphi}_{i}(x, 0)=b_{i}^{1}(x), \quad x \in \bar{B}, \\
\omega(x, 0)=\omega^{0}(x), & \dot{\omega}(x, 0)=\omega^{1}(x), \\
\theta(x, 0)=\theta^{0}(x), & \eta(x, 0)=\eta^{0}(x)
\end{array}
$$


and the following prescribed boundary conditions:

$$
\begin{array}{llll}
u_{i}=\bar{u}_{i} & \text { on } \partial B_{1} \times I, & t_{i}=\bar{t}_{i} & \text { on } \partial B_{1}^{c} \times I, \\
\varphi_{i}=\bar{\varphi}_{i} & \text { on } \partial B_{2} \times I, & m_{i}=\bar{m}_{i} & \text { on } \partial B_{2}^{c} \times I, \\
\omega=\bar{\omega} & \text { on } \partial B_{3} \times I, & \lambda=\bar{\lambda} & \text { on } \partial B_{3}^{c} \times I, \\
\theta=\bar{\theta} & \text { on } \partial B_{4} \times I, & q=\bar{q} & \text { on } \partial B_{4}^{c} \times I,
\end{array}
$$

where $\partial B_{1}, \partial B_{2}, \partial B_{3}$, and $\partial B_{4}$ with respective complements $\partial B_{1}^{c}, \partial B_{2}^{c}, \partial B_{3}^{c}$, and $\partial B_{4}^{c}$ are subsets of the surface $\partial B$ such that

$$
\begin{aligned}
& \partial B_{1} \cap \partial B_{1}^{c}=\partial B_{2} \cap \partial B_{2}^{c}=\partial B_{3} \cap \partial B_{3}^{c}=\partial B_{4} \cap \partial B_{4}^{c}=\emptyset, \\
& \partial B_{1} \cup \partial B_{1}^{c}=\partial B_{2} \cup \partial B_{2}^{c}=\partial B_{3} \cup \partial B_{3}^{c}=\partial B_{4} \cup \partial B_{4}^{c}=\partial B .
\end{aligned}
$$

We assume that:

(1) $a_{i}^{0}, a_{i}^{1}, b_{i}^{0}, b_{i}^{1}, \omega^{0}, \omega^{1}, \theta^{0}$ and $\eta^{0}$ are continuous function on $\bar{B}$;

(2) $\bar{u}_{i}, \bar{\varphi}_{i}, \bar{\omega}$ and $\bar{\theta}$ are continuous function on their domains;

(3) $\bar{t}_{i}, \bar{m}_{i}, \bar{\lambda}$ and $\bar{q}$ are piecewise regular functions in their domains and continuous with regards to the time variable $t$.

By a solution of the mixed initial boundary value problem of the theory thermoelasticity of microstretch bodies in the cylinder $\Omega_{0}=B \times I$ we mean an ordered array $\left(u_{i}, \varphi_{i}, \omega, \theta\right)$ which satisfies (1), (2), and (3) for all $(x, t) \in \Omega_{0}$, the initial conditions (7) and the boundary conditions (8).

\section{Main results}

Let us consider the functions $K$ and $U$ defined on the interval $[0, \infty)$ by the relations

$$
\begin{aligned}
K= & \frac{1}{2} \int_{B}\left(\varrho \dot{u}_{i} \dot{u}_{i}+I_{i j} \dot{\varphi}_{i} \dot{\varphi}_{j}+J \dot{\omega}^{2}\right) d v, \\
U= & \frac{1}{2} \int_{B}\left(A_{i j m n} \varepsilon_{i j} \varepsilon_{m n}+2 B_{i j m n} \varepsilon_{m n} \mu_{i j}+2 D_{i j k} \varepsilon_{i j} \gamma_{k}\right. \\
& \left.+C_{i j m n} \mu_{m n} \mu_{i j}+2 E_{i j k} \mu_{i j} \gamma_{k}+F_{i j} \gamma_{i} \gamma_{j}+d \theta^{2}\right) d \nu .
\end{aligned}
$$

In the next theorem we prove a relationship between the functions $K$ and $U$.

Theorem 1 Assume that the symmetry relations (5) are satisfied. Then we have

$$
\begin{aligned}
U(t)-K(t)= & \frac{1}{2} \int_{0}^{t}[G(t+s, t-s)-G(t-s, t+s)] d s \\
& +\frac{1}{2} \int_{B}\left\{A_{i j m n}(x) \varepsilon_{i j}(x, 2 t) \varepsilon_{m n}(x, 0)\right. \\
& +B_{i j m n}(x)\left[\varepsilon_{i j}(x, 2 t) \mu_{m n}(x, 0)+\varepsilon_{i j}(x, 0) \mu_{m n}(x, 2 t)\right] \\
& +D_{i j k}(x)\left[\varepsilon_{i j}(x, 2 t) \gamma_{k}(x, 0)+\varepsilon_{i j}(x, 0) \gamma_{k}(x, 2 t)\right] \\
& +C_{i j m n}(x) \mu_{i j}(x, 2 t) \mu_{m n}(x, 0) \\
& +E_{i j k}(x)\left[\mu_{i j}(x, 2 t) \gamma_{k}(x, 0)+\mu_{i j}(x, 0) \gamma_{k}(x, 2 t)\right]
\end{aligned}
$$




$$
\begin{aligned}
& \left.+F_{i j}(x) \gamma_{i}(x, 2 t) \gamma_{j}(x, 0)+d(x) \theta(x, 2 t) \theta(x, 0)\right\} d v \\
& -\frac{1}{2} \int_{B}\left[\varrho(x) \dot{u}_{i}(x, 0) \dot{u}_{i}(x, 2 t)+I_{i j} \dot{\varphi}_{i}(x, 0) \dot{\varphi}_{j}(x, 2 t)\right. \\
& +J \dot{\omega}(x, 0) \dot{\omega}(x, 2 t)] d v,
\end{aligned}
$$

where we have denoted by $G(\alpha, \beta)$ the following expression:

$$
\begin{aligned}
G(\alpha, \beta)= & \int_{B}\left[F_{i}(x, \alpha) \dot{u}_{i}(x, \beta)+G_{i}(x, \alpha) \dot{\varphi}_{i}(x, \beta)+L(x, \alpha) \dot{\omega}(x, \beta)-\frac{1}{T_{0}} S(x, \alpha) \dot{\theta}(x, \beta)\right] d v \\
& +\int_{\partial B}\left[t_{i}(x, \alpha) \dot{u}_{i}(x, \beta)+m_{i}(x, \alpha) \dot{\varphi}_{i}(x, \beta)+\lambda(x, \alpha) \dot{\omega}(x, \beta)\right] d a
\end{aligned}
$$

for all $\alpha, \beta \in I$.

Proof Using the constitutive equations (4) and the symmetry relations (5), we obtain

$$
\begin{aligned}
& t_{i j}(t-s) \dot{\varepsilon}_{i j}(t+s)+m_{i j}(t-s) \dot{\mu}_{i j}(t+s)+\lambda_{i}(t-s) \dot{\gamma}_{i}(t+s)+\dot{\eta}(t+s) \theta(t-s) \\
& -\left[t_{i j}(t+s) \dot{\varepsilon}_{i j}(t-s)+m_{i j}(t+s) \dot{\mu}_{i j}(t-s)+\lambda_{i}(t+s) \dot{\gamma}_{i}(t-s)+\dot{\eta}(t-s) \theta(t+s)\right] \\
& =A_{i j m n}(t-s) \varepsilon_{m n}(t-s) \dot{\varepsilon}_{i j}(t+s)+B_{i j m n}(t-s) \mu_{m n}(t-s) \dot{\varepsilon}_{i j}(t+s) \\
& +D_{i j k}(t-s) \gamma_{k}(t-s) \dot{\varepsilon}_{i j}(t+s)+a_{i j}(t-s) \theta(t-s) \dot{\varepsilon}_{i j}(t+s) \\
& +B_{i j m n}(t-s) \varepsilon_{m n}(t-s) \dot{\mu}_{i j}(t+s)+C_{i j m n}(t-s) \mu_{m n}(t-s) \dot{\mu}_{i j}(t+s) \\
& +E_{i j k}(t-s) \gamma_{k}(t-s) \dot{\mu}_{i j}(t+s)+b_{i j}(t-s) \theta(t-s) \dot{\mu}_{i j}(t+s) \\
& +D_{m n i}(t-s) \dot{\gamma}_{i}(t+s) \varepsilon_{m n}(t-s)+E_{m n i}(t-s) \dot{\gamma}_{i}(t+s) \mu_{m n}(t-s) \\
& +F_{i j}(t-s) \gamma_{j}(t-s) \dot{\gamma}_{i}(t+s)+c_{i}(t-s) \theta(t-s) \dot{\gamma}_{i}(t+s) \\
& -a_{i j}(t+s) \dot{\varepsilon}_{i j}(t+s) \theta(t-s)-b_{i j}(t+s) \dot{\mu}_{i j}(t+s) \theta(t-s) \\
& -c_{i}(t+s) \dot{\gamma}_{i}(t+s) \theta(t-s)+d(t+s) \dot{\theta}(t+s) \theta(t-s) \\
& -A_{i j m n}(t+s) \varepsilon_{m n}(t+s) \dot{\varepsilon}_{i j}(t+s) B_{i j m n}(t+s) \mu_{m n}(t+s) \dot{\varepsilon}_{i j}(t-s) \\
& -D_{i j k}(t+s) \gamma_{k}(t+s) \dot{\varepsilon}_{i j}(t-s)-a_{i j}(t+s) \theta(t+s) \dot{\varepsilon}_{i j}(t-s) \\
& -B_{i j m n}(t+s) \varepsilon_{m n}(t+s) \dot{\mu}_{i j}(t+s)-C_{i j m n}(t+s) \mu_{m n}(t+s) \dot{\mu}_{i j}(t-s) \\
& -E_{i j k}(t+s) \gamma_{k}(t+s) \dot{\mu}_{i j}(t-s)-b_{i j}(t+s) \theta(t+s) \dot{\mu}_{i j}(t-s) \\
& -D_{m n i}(t+s) \dot{\gamma}_{i}(t+s) \varepsilon_{m n}(t-s)+E_{m n i}(t+s) \mu_{m n}(t+s) \dot{\gamma}_{i}(t-s) \\
& -F_{i j}(t+s) \gamma_{j}(t+s) \dot{\gamma}_{i}(t-s)-c_{i}(t+s) \theta(t+s) \dot{\gamma}_{i}(t-s) \\
& +a_{i j}(t-s) \dot{\varepsilon}_{i j}(t-s) \theta(t+s)+b_{i j}(t-s) \dot{\mu}_{i j}(t-s) \theta(t+s) \\
& +c_{i}(t-s) \dot{\gamma}_{i}(t-s) \theta(t+s)-d(t-s) \dot{\theta}(t-s) \theta(t+s) \\
& =\frac{\partial}{\partial s}\left[A_{i j m n} \varepsilon_{i j}(t+s) \varepsilon_{m n}(t-s)+2 B_{m n i j} \mu_{m n}(t+s) \varepsilon_{i j}(t-s)\right. \\
& +2 D_{i j k} \gamma_{k}(t+s) \varepsilon_{i j}(t-s)+C_{i j m n} \mu_{i j}(t+s) \mu_{m n}(t-s) \\
& \left.+2 E_{i j k} \gamma_{k}(t+s) \mu_{i j}(t-s)+F_{i j} \gamma_{i}(t+s) \gamma_{j}(t-s)+d \theta(t+s) \theta(t-s)\right] \text {. }
\end{aligned}
$$


Here we have suppressed, for convenience, the dependence of the functions on argument $x$, because there is no likely confusion.

By using the equations of motion (1), the balance of equilibrated forces (2), the energy equation (3) and the geometric equations, we deduce

$$
\begin{aligned}
t_{i j}(t- & s) \dot{\varepsilon}_{i j}(t+s)+m_{i j}(t-s) \dot{\mu}_{i j}(t+s)+\lambda_{i}(t-s) \dot{\gamma}_{i}(t+s)+\dot{\eta}(t+s) \theta(t-s) \\
= & t_{i j}(t-s)\left[\dot{u}_{j, i}(t+s)+\varepsilon_{i j k} \dot{\varphi}_{k}(t+s)\right]+m_{i j}(t-s) \dot{\varphi}_{j, i}(t+s) \\
& +\lambda_{i}(t-s) \dot{\omega}_{, i}(t+s)+\frac{1}{T_{0}} q_{i, i}(t+s) \theta(t-s)+\frac{1}{T_{0}} S(t+s) \theta(t-s) \\
= & {\left[t_{i j}(t-s) \dot{u}_{i}(t+s)+m_{i j}(t-s) \dot{\varphi}_{i}(t+s)+\lambda_{j}(t-s) \dot{\gamma}(t+s)+\frac{1}{T_{0}} q_{j}(t+s) \theta(t-s)\right]_{, j} } \\
& +\varepsilon_{i j k} t_{i j}(t-s) \dot{\varphi}_{k}(t+s)+\frac{1}{T_{0}} S(t+s) \theta(t-s)-t_{i j, j}(t-s) \dot{u}_{i}(t+s) \\
& -m_{i j, j}(t-s) \dot{\varphi}_{i}(t+s)-\lambda_{j, j}(t-s) \dot{\omega}(t+s)-\frac{1}{T_{0}} q_{j}(t+s) \theta_{j, j}(t-s) \\
=[ & \left.t_{i j}(t-s) \dot{u}_{i}(t+s)+m_{i j}(t-s) \dot{\varphi}_{i}(t+s)+\lambda_{j}(t-s) \dot{\gamma}(t+s)+\frac{1}{T_{0}} q_{j}(t+s) \theta(t-s)\right]_{, j} \\
& +\varepsilon_{i j k} t_{i j}(t-s) \dot{\varphi}_{k}(t+s)+\frac{1}{T_{0}} S(t+s) \theta(t-s)+\left[F_{i}(t-s)-\varrho \ddot{u}_{i}(t-s)\right] \dot{u}_{i}(t+s) \\
& +\left[\varepsilon_{i j k} t_{j k}(t-s)+G_{i}(t-s)-I_{i j} \ddot{\varphi}_{j}(t-s)\right] \dot{\varphi}_{i}(t+s) \\
& +[L(t-s)-J \ddot{\omega}(t-s)] \dot{\omega}(t+s)-\frac{1}{T_{0}} k_{i j}(t+s) \theta_{, i}(t+s) \theta_{, j}(t-s) \\
= & \left.t_{i j}(t-s) \dot{u}_{i}(t+s)+m_{i j}(t-s) \dot{\varphi}_{i}(t+s)+\lambda_{j}(t-s) \dot{\gamma}(t+s)+\frac{1}{T_{0}} q_{j}(t+s) \theta(t-s)\right]_{j} \\
& +\varrho \dot{u}_{i}(t-s) \ddot{u}_{i}(t+s)-I_{i j} \dot{\varphi}_{j}(t-s) \ddot{\varphi}_{i}(t+s)-J \dot{\omega}(t-s) \ddot{\omega}(t+s) . \\
& +\frac{1}{T_{0}} S(t+s) \theta(t-s)-\frac{1}{T_{0}} k_{i j}(t+s) \theta_{, i}(t+s) \theta_{j}(t-s) \\
& +\dot{u}_{i}(t+s)+G_{i}(t-s) \dot{\varphi}_{i}(t+s)+L(t-s) \dot{\omega}(t+s)
\end{aligned}
$$

Similarly,

$$
\begin{aligned}
& t_{i j}(t+s) \dot{\varepsilon}_{i j}(t-s)+m_{i j}(t+s) \dot{\mu}_{i j}(t-s)+\lambda_{i}(t+s) \dot{\gamma}_{i}(t-s)+\dot{\eta}(t-s) \theta(t+s) \\
&=[\left.t_{i j}(t+s) \dot{u}_{i}(t-s)+m_{i j}(t+s) \dot{\varphi}_{i}(t-s)+\lambda_{j}(t+s) \dot{\gamma}(t-s)+\frac{1}{T_{0}} q_{j}(t-s) \theta(t+s)\right]_{, j} \\
&+F_{i}(t+s) \dot{u}_{i}(t-s)+G_{i}(t+s) \dot{\varphi}_{i}(t-s)+L(t+s) \dot{\omega}(t-s) \\
&+\frac{1}{T_{0}} S(t-s) \theta(t+s)-\frac{1}{T_{0}} k_{i j}(t-s) \theta_{, i}(t-s) \theta_{, j}(t+s) \\
&+\frac{\partial}{\partial s}\left[\varrho \dot{u}_{i}(t+s) \dot{u}_{i}(t-s)+I_{i j} \dot{\varphi}_{i}(t+s) \dot{\varphi}_{j}(t-s)+J \dot{\omega}(t+s) \dot{\omega}(t-s)\right] \\
& \quad-\varrho \dot{u}_{i}(t-s) \ddot{u}_{i}(t+s)-I_{i j} \dot{\varphi}_{j}(t-s) \ddot{\varphi}_{i}(t+s)-J \dot{\omega}(t-s) \ddot{\omega}(t+s) .
\end{aligned}
$$


Now, we integrate, over $B$, both sides of the equality (12) and then use (5), (13), (14), and the divergence theorem, and it follows that

$$
\begin{aligned}
\int_{B} \frac{\partial}{\partial s} & {\left[A_{i j m n} \varepsilon_{i j}(t+s) \varepsilon_{m n}(t-s)+2 B_{m n i j} \mu_{i j}(t+s) \varepsilon_{m n}(t-s)\right.} \\
& +2 D_{i j k} \gamma_{k}(t+s) \varepsilon_{i j}(t-s)+C_{i j m n} \mu_{i j}(t+s) \mu_{m n}(t-s) \\
& +2 E_{i j k} \gamma_{k}(t+s) \varepsilon_{i j}(t-s)+F_{i j} \gamma_{i}(t+s) \gamma_{j}(t-s) \\
& +d \theta(t+s) \theta(t-s)] d v \\
= & G(t-s, t+s)-G(t+s, t-s) \\
& +\int_{B} \frac{\partial}{\partial s}\left[\varrho \dot{u}_{i}(t-s) \dot{u}_{i}(t+s)+I_{i j} \dot{\varphi}_{j}(t-s) \dot{\varphi}_{i}(t+s)+J \dot{\omega}(t-s) \dot{\omega}(t+s)\right] d v .
\end{aligned}
$$

Finally, we integrate the equality (15) from 0 to $t$ and arrive at the desired result (10) so that the theorem is demonstrated.

In the next lemma we prove another relation between the functions $U$ and $K$ defined by (9).

Lemma 1 Assume that the symmetry relations (5) are satisfied. Then we have the following relations:

$$
\begin{aligned}
2 U(t)= & U(0)+K(0)+\frac{1}{2} \int_{B}\left\{A_{i j m n} \varepsilon_{i j}(2 t) \varepsilon_{m n}(0)+B_{m n i j}\left[\varepsilon_{m n}(2 t) \mu_{i j}(0)+\varepsilon_{m n}(0) \mu_{i j}(2 t)\right]\right. \\
& +D_{i j k}\left[\gamma_{k}(2 t) \varepsilon_{i j}(0)+\gamma_{k}(0) \varepsilon_{i j}(2 t)\right]+C_{i j m n} \mu_{i j}(2 t) \mu_{m n}(0) \\
& \left.+E_{i j k}\left[\gamma_{k}(2 t) \mu_{i j}(0)+\gamma_{k}(0) \mu_{i j}(2 t)\right]+F_{i j} \gamma_{i}(2 t) \gamma_{j}(0)+d \theta(2 t) \theta(0)\right\} d v \\
& -\frac{1}{2} \int_{B}\left[\varrho \dot{u}_{i}(2 t) \dot{u}_{i}(0)+I_{i j} \dot{\varphi}_{j}(2 t) \dot{\varphi}_{i}(0)+J \dot{\omega}(2 t) \dot{\omega}(0)\right] d v \\
& +\frac{1}{2} \int_{0}^{t}[G(t+s, t-s)-G(t-s, t+s)+2 P(s)] d s-\frac{1}{T_{0}} \int_{0}^{t} \int_{B} k_{i j} \theta_{j} \theta_{, i} d v d s, \\
2 K(t)= & U(0)+K(0)-\frac{1}{2} \int_{B}\left\{A_{i j m n} \varepsilon_{i j}(2 t) \varepsilon_{m n}(0)+B_{m n i j}\left[\varepsilon_{m n}(2 t) \mu_{i j}(0)+\varepsilon_{m n}(0) \mu_{i j}(2 t)\right]\right. \\
& +D_{i j k}\left[\gamma_{k}(2 t) \varepsilon_{i j}(0)+\gamma_{k}(0) \varepsilon_{i j}(2 t)\right]+C_{i j m n} \mu_{i j}(2 t) \mu_{m n}(0) \\
& \left.+E_{i j k}\left[\gamma_{k}(2 t) \mu_{i j}(0)+\gamma_{k}(0) \mu_{i j}(2 t)\right]+F_{i j} \gamma_{i}(2 t) \gamma_{j}(0)+d \theta(2 t) \theta(0)\right\} d v \\
& -\frac{1}{2} \int_{B}\left[\varrho \dot{u}_{i}(2 t) \dot{u}_{i}(0)+I_{i j} \dot{\varphi}_{j}(2 t) \dot{\varphi}_{i}(0)+J \dot{\omega}(2 t) \dot{\omega}(0)\right] d v \\
& -\frac{1}{2} \int_{0}^{t}[G(t+s, t-s)-G(t-s, t+s)-2 P(s)] d s-\frac{1}{T_{0}} \int_{0}^{t} \int_{B} k_{i j} \theta_{, j} \theta_{, i} d v d s
\end{aligned}
$$

for all $t \in[0, \infty)$.

The function $P$ is defined by

$$
\begin{aligned}
P(t)= & \int_{B}\left(F_{i} \dot{u}_{i}+G_{i} \dot{\varphi}_{i}+L \dot{\omega}+\frac{1}{T_{0}} S \theta\right) d v \\
& +\int_{\partial B}\left(t_{i} \dot{u}_{i}+m_{i} \dot{\varphi}_{i}+\lambda \dot{\omega}+\frac{1}{T_{0}} q \theta\right) d a .
\end{aligned}
$$


Proof Taking into account the equations of motion (1), the balance of equilibrated forces (2), the energy equation (3), and the constitutive equations (4), we obtain

$$
\begin{aligned}
A_{i j m n} \varepsilon_{m n} \dot{\varepsilon}_{i j}+B_{i j m n}\left(\mu_{m n} \dot{\varepsilon}_{i j}+\dot{\mu}_{m n} \varepsilon_{i j}\right)+D_{i j k}\left(\gamma_{k} \dot{\varepsilon}_{i j}+\dot{\gamma}_{k} \varepsilon_{i j}\right) & +C_{i j m n} \mu_{m n} \dot{\mu}_{i j}+E_{i j k}\left(\gamma_{k} \dot{\mu}_{i j}+\dot{\gamma}_{k} \mu_{i j}\right)+F_{i j} \gamma_{i} \dot{\gamma}_{j}+d \theta \dot{\theta} \\
= & t_{i j} \dot{\varepsilon}_{i j}+m_{i j} \dot{\mu}_{i j}+\lambda_{i} \dot{\gamma}_{i}+\dot{\eta} \theta \\
= & \left(t_{i j} \dot{u}_{i}+m_{i j} \dot{\varphi}_{i}+\lambda_{j} \dot{\omega}+\frac{1}{T_{0}} q_{j} \theta\right)_{, j} \\
& +F_{i} \dot{u}_{i}+G_{i} \dot{\varphi}_{i}+L \dot{\omega}+\frac{1}{T_{0}} S \theta \\
& -\varrho \dot{u}_{i} \ddot{u}_{i}-I_{i j} \dot{\varphi}_{j} \ddot{\varphi}_{i}-J \dot{\omega} \ddot{\omega}-\frac{1}{T_{0}} k_{i j} \theta_{j j} \theta_{, i} .
\end{aligned}
$$

From this equality it is easy to deduce that

$$
\dot{U}+\dot{K}=P-\frac{1}{T_{0}} \int_{B} k_{i j} \theta_{, j} \theta_{, i} d v .
$$

By integrating this relation over the interval $[0, t]$ we obtain

$$
\begin{aligned}
U(t)+K(t)= & U(0)+K(0)+\int_{0}^{t} P(s) d s \\
& -\frac{1}{T_{0}} \int_{0}^{t} \int_{B} k_{i j} \theta_{j} \theta_{, i} d v d s, \quad \forall t \in[0, \infty) .
\end{aligned}
$$

If we add (10) and (19), term by term, (16) follows.

If we subtract (10) from (19), term by term, (17) follows and this concludes Lemma 1.

The uniqueness result from the next theorem is based on the results from Theorem 1 and Lemma 1.

\section{Theorem 2 Assume that:}

(i) the symmetry relations (5) are satisfied;

(ii) $\varrho, I_{i j}$ and $J$ are strictly positive;

(iii) $d$ is strictly positive or strictly negative;

(iv) the conductivity tensor $k_{i j}$ is positive semi-definite.

Then the mixed initial boundary value problem of thermoelasticity of microstretch materials consisting of (1)-(3), the initial condition (7), and the boundary condition (8) has at most one solution.

Proof Suppose, to the contrary, that our mixed problem has two solutions,

$$
\left(u_{i}^{1}, \varphi_{i}^{1}, \omega^{1}, \theta^{1}\right), \quad\left(u_{i}^{2}, \varphi_{i}^{2}, \omega^{2}, \theta^{2}\right) .
$$

Let us denote by

$$
\left(u_{i}, \varphi_{i}, \omega, \theta\right)
$$


the difference of two solutions, where

$$
u_{i}=u_{i}^{1}-u_{i}^{2}, \quad \varphi_{i}=\varphi_{i}^{1}-\varphi_{i}^{2}, \quad \omega=\omega^{1}-\omega^{2}, \quad \theta=\theta^{1}-\theta^{2} .
$$

Because of linearity, this difference is also a solution of our problem, but it corresponds to null data.

Thus, from (17) we obtain

$$
\begin{aligned}
\int_{B}\left[\varrho \dot{u}_{i}(t) \dot{u}_{i}(t)+I_{i j} \dot{\varphi}_{j}(t) \dot{\varphi}_{i}(t)+J \dot{\omega}^{2}(t)\right] d v \\
+\frac{1}{T_{0}} \int_{0}^{t} \int_{B} k_{i j} \theta_{, j} \theta_{, i} d v d s=0, \quad \forall t \in[0, \infty) .
\end{aligned}
$$

By using the hypotheses (ii) and (iv) of the theorem, (20) implies that

$$
\dot{u}_{i}=0, \quad \dot{\varphi}_{i}=0, \quad \dot{\omega}=0 \quad \text { on } B \times I
$$

and

$$
\int_{0}^{t} \int_{B} k_{i j} \theta_{, j} \theta_{, i} d \nu d s=0, \quad \forall t \in[0, \infty)
$$

But $u_{i}, \varphi_{i}$, and $\omega$ vanish initially, so that from (21) we deduce

$$
u_{i}=0, \quad \varphi_{i}=0, \quad \omega=0 \quad \text { on } B \times I .
$$

Taking into account (22) and (23), (16) reduces to

$$
\int_{B} d \theta^{2} d v=0
$$

Since $d>0$ or $d<0$, this relation yields

$$
\theta=0 \quad \text { on } B \times I \text {. }
$$

From (23) and (24) we deduce that the difference of the two solutions is null, i.e., we have the uniqueness of solution and Theorem 2 is demonstrated.

Consider two scalar functions $u$ and $v$ which are defined on $B \times I$ and continuous with respect to time.

As is well known, the convolution product of the function $u$ and $v$ is defined by the integral

$$
(u * v)(x, t)=\int_{0}^{t} u(x, t-s) v(x, s) d s, \quad(x, t) \in B \times I .
$$

Also, let us consider the functions $l(t)$ and $g(t)$ defined by

$$
l(t)=1, \quad g(t)=(l * l)(t)=t, \quad t \in I .
$$


For a continuous function $h$ defined on $B \times I$, we denote by $\bar{h}(x, t)$ the convolution product $l * h$, i.e.

$$
\bar{h}(x, t)=\int_{0}^{t} h(x, s) d s .
$$

Using these considerations, we can write the energy equation (3) and the initial condition

$$
\eta(x, 0)=\eta_{0}(x)
$$

in the equivalent form

$$
T_{0} \eta=\bar{q}_{i, i}+W
$$

where

$$
W=\bar{S}+T_{0} \eta_{0} .
$$

Consider two external data systems $L^{(\alpha)}$, which act on the thermoelastic-microstretch material, defined by

$$
\begin{aligned}
L^{(\alpha)}= & \left\{F_{i}^{(\alpha)}, G_{i}^{(\alpha)}, L^{(\alpha)}, S^{(\alpha)}, \tilde{u}_{i}^{(\alpha)}, \tilde{\varphi}_{i}^{(\alpha)}, \tilde{\omega}^{(\alpha)}, \tilde{t}_{i}^{(\alpha)}, \tilde{m}_{i}^{(\alpha)}, \tilde{\lambda}^{(\alpha)}, \tilde{\theta}^{(\alpha)}, \tilde{q}^{(\alpha)},\right. \\
& \left.a_{i}^{0(\alpha)}, a_{i}^{1(\alpha)}, b_{i}^{0(\alpha)}, b_{i}^{1(\alpha)}, \omega^{0(\alpha)}, \omega^{1(\alpha)}, \theta^{0(\alpha)}, \eta_{0}^{(\alpha)}\right\}, \quad \alpha=1,2,
\end{aligned}
$$

and denote by $p^{(\alpha)}$ a solution of the mixed problem which corresponds to $L^{(\alpha)}$

$$
p^{(\alpha)}=\left\{u_{i}^{(\alpha)}, \varphi_{i}^{(\alpha)}, \omega^{(\alpha)}, \theta^{(\alpha)}\right\} .
$$

Also, we use the following notations:

$$
\begin{aligned}
& t_{i}^{(\alpha)}=t_{i j}^{(\alpha)} n_{j}, \quad m_{i}^{(\alpha)}=m_{i j}^{(\alpha)} n_{j}, \quad \lambda^{(\alpha)}=\lambda_{i}^{(\alpha)} n_{i}, \\
& q^{(\alpha)}=q_{i}^{(\alpha)} n_{j}, \quad W^{(\alpha)}=l * S^{(\alpha)}+T_{0} \eta_{0}^{(\alpha)} .
\end{aligned}
$$

Lemma 2 Assume that the symmetry relations (5) are satisfied. Consider the functions $E_{\alpha \beta}(t, \tau)$ defined by

$$
\begin{aligned}
E_{\alpha \beta}(t, \tau)= & \int_{\partial B}\left[t_{i}^{(\alpha)}(t) u_{i}^{(\beta)}(\tau)+m_{i}^{(\alpha)}(t) \varphi_{i}^{(\beta)}(\tau)+\lambda^{(\alpha)}(t) \omega^{(\beta)}(\tau)-\frac{1}{T_{0}} \bar{q}^{(\alpha)}(t) \theta^{(\beta)}(\tau)\right] d a \\
& +\int_{B}\left[F_{i}^{(\alpha)}(t) u_{i}^{(\beta)}(\tau)+G_{i}^{(\alpha)}(t) \varphi_{i}^{(\beta)}(\tau)+L^{(\alpha)}(t) \omega^{(\beta)}(\tau)\right. \\
& \left.-\frac{1}{T_{0}} W^{(\alpha)}(t) \theta^{(\beta)}(\tau)\right] d v \\
& -\int_{B}\left[\varrho \ddot{u}_{i}^{(\alpha)}(t) \dot{u}_{i}^{(\beta)}(\tau)+I_{i j} \ddot{\varphi}_{j}^{(\alpha)}(t) \dot{\varphi}_{i}^{(\beta)}(\tau)+J \ddot{\omega}^{(\alpha)}(t) \dot{\omega}^{(\beta)}(\tau)\right] d v \\
& +\frac{1}{T_{0}} \int_{B} \bar{q}_{i}^{(\alpha)}(t) \theta_{, i}^{(\beta)}(\tau) d v, \quad \forall t, \tau \in I .
\end{aligned}
$$


Then we have

$$
E_{\alpha \beta}(t, \tau)=E_{\beta \alpha}(t, \tau)
$$

for all $t, \tau \in I$.

Proof We introduce the notation

$$
I_{\alpha \beta}(t, \tau)=t_{i j}^{(\alpha)}(t) \varepsilon_{i j}^{(\beta)}(\tau)+m_{i j}^{(\alpha)}(t) \mu_{i j}^{(\beta)}(\tau)+\lambda_{i}^{(\alpha)}(t) \gamma_{i}^{(\beta)}(\tau)-\eta^{(\alpha)}(t) \theta^{(\beta)}(\tau) .
$$

From (32) and the constitutive equations (4) we deduce

$$
\begin{aligned}
I_{\alpha \beta}(t, \tau)= & A_{i j m n} \varepsilon_{m n}^{(\alpha)}(t) \varepsilon_{i j}^{(\beta)}(\tau)+B_{m n i j}\left[\mu_{m n}^{(\alpha)}(t) \varepsilon_{i j}^{(\beta)}(\tau)+\mu_{m n}^{(\beta)}(t) \varepsilon_{i j}^{(\alpha)}(\tau)\right] \\
& +D_{i j k}\left[\gamma_{k}^{(\alpha)}(t) \varepsilon_{i j}^{(\beta)}(\tau)+\gamma_{k}^{(\beta)}(t) \varepsilon_{i j}^{(\alpha)}(\tau)\right]+C_{i j m n} \mu_{m n}^{(\alpha)}(t) \mu_{i j}^{(\beta)}(\tau) \\
& +E_{i j k}\left[\gamma_{k}^{(\alpha)}(t) \mu_{i j}^{(\beta)}(\tau)+\gamma_{k}^{(\beta)}(t) \mu_{i j}^{(\alpha)}(\tau)\right] \\
& +F_{i j} \gamma_{i}^{(\alpha)}(t) \gamma_{j}^{(\beta)}(\tau)-d \theta^{(\alpha)}(t) \theta^{(\beta)}(\tau) .
\end{aligned}
$$

Using the symmetry relations (5), from (33) we obtain

$$
I_{\alpha \beta}(t, \tau)=I_{\beta \alpha}(t, \tau) .
$$

On the other hand, using the equations of motion (1), the balance of equilibrated forces (2), and the energy equation (3) in (27) and (32), we are lead to the following expression of $I_{\alpha \beta}(t, \tau)$ :

$$
\begin{aligned}
I_{\alpha \beta}(t, \tau)= & {\left[t_{i j}^{(\alpha)}(t) u_{i}^{(\beta)}(\tau)+m_{i j}^{(\alpha)}(t) \varphi_{i}^{(\beta)}(\tau)+\lambda_{j}^{(\alpha)}(t) \omega^{(\beta)}(\tau)-\frac{1}{T_{0}} \bar{q}_{j}^{(\alpha)}(t) \theta^{(\beta)}(\tau)\right]_{, j} } \\
& +F_{i}^{(\alpha)}(t) u_{i}^{(\beta)}(\tau)+G_{i}^{(\alpha)}(t) \varphi_{i}^{(\beta)}(\tau)+L^{(\alpha)}(t) \omega^{(\beta)}(\tau)-\frac{1}{T_{0}} W^{(\alpha)}(t) \theta^{(\beta)}(\tau) \\
& -\varrho \ddot{u}_{i}^{(\alpha)}(t) u_{i}^{(\beta)}(\tau)-I_{i j} \ddot{\varphi}_{j}^{(\alpha)}(t) \varphi_{i}^{(\beta)}(\tau)-J \ddot{\omega}^{(\alpha)}(t) \omega^{(\beta)}(\tau)+\frac{1}{T_{0}} \bar{q}_{i}^{(\alpha)}(t) \theta_{, i}^{(\beta)}(\tau) .
\end{aligned}
$$

Now, we integrate (35) over $B$, then we use the symmetry relations (34) and the divergence theorem so that we obtain the desired result (31) and Lemma 2 is proved.

Based on the result of Lemma 2 we can prove the reciprocal result from the next theorem.

Theorem 3 Assume that the symmetry relations (5) are satisfied. Let $p^{(\alpha)}$ be a solution corresponding to the external data $L^{(\alpha)}, \alpha=1,2$. Then the following reciprocal relation is valid:

$$
\begin{array}{rl}
\int_{\partial B} g & *\left[t_{i}^{(1)} * u_{i}^{(2)}+m_{i}^{(1)} * \varphi_{i}^{(2)}+\lambda^{(1)} * \omega^{(2)}-\frac{1}{T_{0}} l * q^{(1)} * \theta^{(2)}\right] d a \\
& +\int_{B}\left[\mathcal{F}_{i}^{(1)} * u_{i}^{(2)}+\mathcal{G}_{i}^{(1)} * \varphi_{i}^{(2)}+\mathcal{L}^{(1)} * \omega^{(2)}-\frac{1}{T_{0}} g * W^{(1)} * \theta^{(2)}\right] d v
\end{array}
$$




$$
\begin{aligned}
= & \int_{\partial B} g *\left[t_{i}^{(2)} * u_{i}^{(1)}+m_{i}^{(2)} * \varphi_{i}^{(1)}+\lambda^{(2)} * \omega^{(1)}-\frac{1}{T_{0}} l * q^{(2)} * \theta^{(1)}\right] d a \\
& +\int_{B}\left[\mathcal{F}_{i}^{(2)} * u_{i}^{(1)}+\mathcal{G}_{i}^{(2)} * \varphi_{i}^{(1)}+\mathcal{L}^{(2)} * \omega^{(1)}-\frac{1}{T_{0}} g * W^{(2)} * \theta^{(1)}\right] d \nu
\end{aligned}
$$

where we have used the notations

$$
\begin{aligned}
& \mathcal{F}_{i}^{(\alpha)}=g * F_{i}^{(\alpha)}+\varrho\left(t a_{i}^{1(\alpha)}+a_{i}^{0(\alpha)}\right), \\
& \mathcal{G}_{i}^{(\alpha)}=g * G_{i}^{(\alpha)}+I_{i j}\left(t b_{j}^{1(\alpha)}+b_{j}^{0(\alpha)}\right), \\
& \mathcal{L}_{i}^{(\alpha)}=g * L^{(\alpha)}+J\left(t w^{1(\alpha)}+w^{0(\alpha)}\right) .
\end{aligned}
$$

Proof We use the substitution $t=s$ and $\tau=r-s$ in (31) and integrate the resulting relation on the interval $[0, s]$ such that, by using the symmetry relations $(5)$, we obtain

$$
\begin{aligned}
\int_{\partial B}[ & \left.t_{i}^{(1)} * u_{i}^{(2)}+m_{i}^{(1)} * \varphi_{i}^{(2)}+\lambda^{(1)} * \omega^{(2)}-\frac{1}{T_{0}} l * q^{(1)} * \theta^{(2)}\right] d a \\
& +\int_{B}\left[F_{i}^{(1)} * u_{i}^{(2)}+G_{i}^{(1)} * \varphi_{i}^{(2)}+L^{(1)} * \omega^{(2)}-\frac{1}{T_{0}} W^{(1)} * \theta^{(2)}\right] d \nu \\
& -\int_{B}\left[\varrho \ddot{u}_{i}^{(1)} u_{i}^{(2)}+I_{i j} \ddot{\varphi}_{i}^{(1)} \varphi_{j}^{(2)}+J \ddot{\omega}^{(1)} \omega^{(2)}\right] d v \\
= & \int_{\partial B}\left[t_{i}^{(2)} * u_{i}^{(1)}+m_{i}^{(2)} * \varphi_{i}^{(1)}+\lambda^{(2)} * \omega^{(1)}-\frac{1}{T_{0}} l * q^{(2)} * \theta^{(1)}\right] d a \\
& +\int_{B}\left[F_{i}^{(2)} * u_{i}^{(1)}+G_{i}^{(2)} * \varphi_{i}^{(1)}+L^{(2)} * \omega^{(1)}-\frac{1}{T_{0}} W^{(2)} * \theta^{(1)}\right] d \nu \\
& -\int_{B}\left[\varrho \ddot{u}_{i}^{(2)} u_{i}^{(1)}+I_{i j} \ddot{\varphi}_{i}^{(2)} \varphi_{j}^{(1)}+J \ddot{\omega}^{(2)} \omega^{(1)}\right] d \nu .
\end{aligned}
$$

It is easy to prove that

$$
\begin{aligned}
& g * \ddot{u}_{i}^{(\alpha)}=u_{i}^{(\alpha)}-t a_{i}^{1(\alpha)}-a_{i}^{0(\alpha)}, \\
& g * \ddot{\varphi}_{i}^{(\alpha)}=\varphi_{i}^{(\alpha)}-t b_{i}^{1(\alpha)}-b_{i}^{0(\alpha)}, \\
& g * \ddot{\omega}^{(\alpha)}=\omega^{(\alpha)}-t w^{1(\alpha)}-w^{0(\alpha)} .
\end{aligned}
$$

Taking the convolution of (38) with $g$ and using (39), we obtain the reciprocal relation (36) and the proof of Theorem 3 is complete.

Remark In the case of null boundary data, from (36) we deduce that the operator of the thermoelastodynamics of microstretch bodies is symmetric with regard to the convolution.

Based on this symmetry, we can obtain some variational theorems of Gurtin type in classical thermoelasticity.

Also, based on the symmetry relations (31) we can obtain a minimum principle similar to those obtained by Reiss [19] in the classical isothermal case. 
Theorem 4 Assume that the symmetry relations (5) are satisfied. Let us consider the function

$$
\begin{aligned}
H(r, s)= & \int_{B}\left[F_{i}(r) u_{i}(s)+G_{i}(r) \varphi_{i}(s)+L(r) w(s)-\frac{1}{T_{0}} w(r) \theta(s)\right] d v \\
& +\int_{\partial B}\left[t_{i}(r) u_{i}(s)+m_{i}(r) \varphi_{i}(s)+\lambda(r) w(s)-\frac{1}{T_{0}} \bar{q}(r) \theta(s)\right] d a
\end{aligned}
$$

for all $r, s \in I$.

Then we have

$$
\begin{aligned}
& \frac{d}{d t}\left[\int_{B}\left(\varrho u_{i} u_{i}+I_{i j} \varphi_{i} \varphi_{j}+J \omega^{2}\right) d v+\frac{1}{T_{0}} \int_{0}^{t} \int_{B} k_{i j} \theta_{j} \theta_{, i} d v d s\right] \\
& =\int_{0}^{t}[H(t-s, t+s)-H(t+s, t-s)] d s \\
& \quad+\int_{B}\left\{\varrho\left[\dot{u}_{i}(2 t) u_{i}(0)+\dot{u}_{i}(0) u_{i}(2 t)\right]+I_{i j}\left[\dot{\varphi}_{i}(2 t) \varphi_{j}(0)+\dot{\varphi}_{i}(0) \varphi_{j}(2 t)\right]\right. \\
& \left.\quad+J\left[\dot{\omega}(2 t) \omega_{j}(0)+\dot{\omega}(0) \omega(2 t)\right]\right\} d v .
\end{aligned}
$$

Proof Using the result on $E_{\alpha \beta}$ from Lemma 2, we obtain

$$
\int_{0}^{t} E_{11}(t+s, t-s) d s=\int_{0}^{t} E_{11}(t-s, t+s) d s .
$$

Let us apply (42) to the process

$$
\left(u_{i}, \varphi_{i}, \omega, \theta, t_{i} m_{i}, \lambda, \eta, q\right) .
$$

From (30) and (40), we obtain the equality

$$
\begin{aligned}
& \int_{0}^{t} E_{11}(t+s, t-s) d s \\
& =\int_{0}^{t} H_{11}(t+s, t-s) d s \\
& \quad-\int_{0}^{t} \int_{B}\left[\varrho \ddot{u}_{i}(t+s) u_{i}(t-s)+I_{i j} \ddot{\varphi}_{i}(t+s) \varphi_{j}(t-s)+J \ddot{\omega}(t+s) \omega(t-s)\right] d v d s \\
& \quad+\frac{1}{T_{0}} \int_{0}^{t} \int_{B} k_{i j} \theta_{, j}(t+s) \theta_{, i}(t-s) d v d s .
\end{aligned}
$$

Similarly,

$$
\begin{aligned}
& \int_{0}^{t} E_{11}(t-s, t+s) d s \\
& =\int_{0}^{t} H_{11}(t-s, t+s) d s \\
& \quad-\int_{0}^{t} \int_{B}\left[\varrho \ddot{u}_{i}(t-s) u_{i}(t+s)+I_{i j} \ddot{\varphi}_{i}(t-s) \varphi_{j}(t+s)+J \ddot{\omega}(t-s) \omega(t+s)\right] d v d s \\
& \quad+\frac{1}{T_{0}} \int_{0}^{t} \int_{B} k_{i j} \theta_{, j}(t-s) \theta_{, i}(t+s) d v d s .
\end{aligned}
$$


It is easy to prove the relations

$$
\begin{aligned}
& \int_{0}^{t} \ddot{f}(t+s) h(t-s) d s=\dot{f}(2 t) h(0)-\dot{f}(t) h(t)+\int_{0}^{t} \dot{h}(t-s) \dot{f}(t+s) d s, \\
& \int_{0}^{t} \ddot{h}(t-s) f(t+s) d s=\dot{h}(t) f(t)-\dot{h}(0) f(2 t)+\int_{0}^{t} \dot{h}(t-s) \dot{f}(t+s) d s, \\
& \int_{0}^{t} f(t+s) \dot{h}(t-s) d s=-h(0) f(2 t)+f(t) h(t)+\int_{0}^{t} \dot{f}(t+s) h(t-s) d s .
\end{aligned}
$$

Using the symmetry relations (5) and (45), from (42), (43), and (44) we obtain the desired result (41) and the proof of Theorem 4 is complete.

A similar reciprocal result has been obtained in [11], but using some strong hypotheses on the thermoelastic coefficients.

If the conductivity tensor $k_{i j}$ is assumed be positive definite, then the result of Theorem 4 can be used to obtain the uniqueness result established in [18].

Now, our intention is to give another proof of Theorem 2 by using the result of Theorem 4. Let us consider that the mixed problem formulated above has two solutions,

$$
\left(u_{i}^{1}, \varphi_{i}^{1}, \omega^{1}, \theta^{1}\right), \quad\left(u_{i}^{2}, \varphi_{i}^{2}, \omega^{2}, \theta^{2}\right) .
$$

Let us denote by

$$
\left(u_{i}, \varphi_{i}, \omega, \theta\right)
$$

the difference of two solutions, where

$$
u_{i}=u_{i}^{1}-u_{i}^{2}, \quad \varphi_{i}=\varphi_{i}^{1}-\varphi_{i}^{2}, \quad \omega=\omega^{1}-\omega^{2}, \quad \theta=\theta^{1}-\theta^{2} .
$$

If we apply (41) for the difference, we are lead to

$$
\int_{B}\left(\varrho u_{i} u_{i}+I_{i j} \varphi_{i} \varphi_{j}+J \omega^{2}\right) d v+\frac{1}{T_{0}} \int_{0}^{t} \int_{B} k_{i j} \theta_{, j} \theta_{, i} d \nu d s=0 .
$$

From this equality, by using the hypotheses (ii) and (iv) of Theorem 2, we obtain

$$
u_{i}=0, \quad \varphi_{i}=0, \quad \omega=0 .
$$

Then, from (16), (17), and hypothesis (iii) of Theorem 2, we obtain

$$
\theta=0 \quad \text { on } B \times I \text {, }
$$

which proves Theorem 4 .

Remark Using a similar procedure as in [18] and [9], we can use (16), (17), and (41) to obtain some continuous dependence results. 


\section{Concluding remarks}

The intrinsic rotations, microstructural expansion, and contractions do not affect the existence nor the uniqueness and continuous dependence of the solution of the mixed initial boundary value problem for thermoelastic bodies.

Also, it is not necessary to constrain the conductivity tensor to be positive definite to obtain the basic results of the theory of thermoelasticity of microstretch materials. It is sufficient for this tensor to be positive semi-defined as results from the Clausius-Duhem inequality.

\section{Competing interests}

The authors declare that they have no competing interests.

\section{Authors' contributions}

MIM proposed main results of the paper and verified all calculations and demonstrations. RPA proposed the method of demonstration of results, without using a sophisticated mathematical apparatus. Also, he controlled the final shape of the paper. IAA performed all calculations and demonstrations and took into account the suggestions given by MIM. All authors read and approved the final manuscript.

\section{Author details}

${ }^{1}$ Department of Mathematics, Transilvania University of Brasov, Brasov, Romania. ${ }^{2}$ Department of Mathematics, Texas A\&M University-Kingsville, Kingsville, USA. ${ }^{3}$ Department of Mathematics, Faculty of Science and Arts-Khulais, King Abdulaziz University, Jeddah, Saudi Arabia. ${ }^{4}$ Department of Mathematics, Faculty of Science, Sohag University, Sohag, Egypt.

\section{Acknowledgements}

We express our gratitude to the referees for their valuable criticisms of the manuscript and for helpful suggestions.

Received: 26 February 2014 Accepted: 12 May 2014 Published: 22 May 2014

\section{References}

1. Eringen, AC: Theory of micromorphic materials with memory. Int. J. Eng. Sci. 10, 623-641 (1972)

2. Eringen, AC: Theory of thermo-microstretch elastic solids. Int. J. Eng. Sci. 28, 1291-1301 (1990)

3. Ciarletta, M: On the bending of microstretch elastic plates. Int. J. Eng. Sci. 37, 1309-1318 (1995)

4. Ciarletta, M, Svanadze, M, Buonanno, L: Plane waves and vibrations in the theory of micropolar thermoelasticity for materials with voids. Eur. J. Mech. A, Solids 28, 897-903 (2009)

5. lesan, D, Pompei, A: Equilibrium theory of microstretch elastic solids. Int. J. Eng. Sci. 33, 399-410 (1995)

6. Agarwal, RP, O'Regan, D, Lashmikantham, V, Leela, S: A generalized upper and lower solution method for singular initial value problems. Comput. Math. Appl. 47, 739-750 (2004)

7. Agarwal, RP, O'Regan, D: Boundary value problems on the half line in the theory of colloids. Math. Probl. Eng. 8, 143-150 (2002)

8. Marin, M: A partition of energy in thermoelasticity of microstretch bodies. Nonlinear Anal., Real World Appl. 11(4), 2436-2447 (2010)

9. Marin, M: Lagrange identity for microstretch thermoelastic materials. J. Math. Anal. Appl. 361(1), $275-286$ (2010)

10. Marin, M: Some estimates on vibrations in thermoelasticity of dipolar bodies. J. Vib. Control 16(1), 33-47 (2010)

11. Marin, M: An evolutionary equation in thermoelasticity of dipolar bodies. J. Math. Phys. 40(3), 1391-1399 (1999)

12. Marin, M, Agarwal, RP, Mahmoud, SR: Modeling a microstretch thermoelastic body with two temperatures. Abstr. Appl. Anal. 2013, Article ID 583464 (2013). doi:10.1155/2013/583464

13. Abd-Alla, AM, Mahmoud, SR, Abo-Dahab, SM, Helmy, Ml: Propagation of S-wave in a non-homogeneous anisotropic incompressible and initially stressed medium under influence of gravity field. Appl. Math. Comput. 217(9), 4321-4332 (2011)

14. Abd-alla, AM, Abbas, IA: Magnetoelastic longitudinal wave propagation in a transversely isotropic circular cylinder. Appl. Math. Comput. 127(2-3), 347-360 (2002)

15. Othman, MIA: Effect of rotation on plane waves in generalized thermoelasticity with two relaxation times. Int. J. Solids Struct. 41, 2939-2956 (2004)

16. Othman, MIA, Singh, B: The effect of rotation on generalized micropolar thermoelasticity for a half-space under five theories. Int. J. Solids Struct. 44(9), 2748-2762 (2007)

17. Boschi, E, Mainardi, F: Body loadings in thermo-microstretch elastic solids. Geophys. J. R. Astron. Soc. 34, 313-320 (1973)

18. Knops, RJ, Payne, LE: On uniqueness and continuous dependence in dynamical problems of thermoelasticity. Int J. Solids Struct. 6, 1173-1184 (1970)

19. Reiss, R: Minimum principles for linear elastodynamics. J. Elast. 8(1), 35-45 (1978)

10.1186/1687-2770-2014-129

Cite this article as: Marin et al.: Effect of intrinsic rotations, microstructural expansion and contractions in initial boundary value problem of thermoelastic bodies. Boundary Value Problems 2014, 2014:129 